\title{
THE GROUP OF HOMOTOPY EQUIVALENCES OF A SPACE ${ }^{1}$
}

\author{
BY M. ARKOWITZ AND C. R. CURJEL
}

Communicated by Deane Montgomery, December 2, 1963

1. Introduction. Let $\varepsilon(X)$ denote the collection of homotopy classes of homotopy equivalences of a space $X$ with itself. Composition of maps induces a group structure in $\varepsilon(X)$. From the point of view of categories $\mathcal{E}(X)$ is the group of equivalences of the object $X$ in the category of spaces and homotopy classes of maps. Thus it is the homotopy analog of the automorphism group of a group and the group of homeomorphisms of a space.

In this note we present some theorems which relate properties of the homotopy groups of $X$ to algebraic properties of $\varepsilon(X)$. In such a study one encounters the difficulties associated with the problem of composing homotopy classes of maps. In addition one can easily show that for any finite group $T$ there exists a finite complex $X$ such that $\mathcal{E}(X)$ contains $T$ as a subgroup. Thus any group theoretic property which does not hold for all finite groups cannot be true for all groups $\mathcal{E}(X)$.

The hypotheses of all of our theorems are not intricate, and thus our results provide specific information on $\varepsilon(X)$ for many $X$. $\$ 2$ contains theorems on the group of equivalences of any 1-connected finite complex, and $\$ 3$ deals with associative $H$-spaces. At the end of each section we give a brief description of our methods. Details and applications will appear elsewhere.

Various results on $\mathcal{E}(X)$ have been obtained by Barcus-Barratt $[1, \S 6]$, P. Olum (to appear) and D. W. Kahn (to appear). Furthermore, W. Shih [5] has constructed a spectral sequence for $\mathcal{E}(X)$.

We should like to thank R. P. Langlands for several discussions on Proposition 9.

2. General theorems. We consider only 1-connected spaces of the homotopy type of a CW-complex with finitely generated homotopy groups in all dimensions. Let $X^{(n)}$ be an $n$th Postnikov section of $X$. A straightforward obstruction argument yields

Lemma 1. If $X$ is a finite $\mathrm{CW}$-complex then $\mathcal{E}(X) \approx \mathcal{E}\left(X^{(n)}\right)$ for all $n>\operatorname{dim} X$.

${ }^{1}$ Research supported by the U. S. Air Force Office of Scientific Research and the National Science Foundation (NSF-GP779). 
By assigning to an element of $\mathcal{E}(X)$ its induced homotopy automorphism, one obtains a homomorphism $I: \mathcal{E}(X) \rightarrow \coprod_{k}$ Aut $\pi_{k}(X)$ whose kernel is denoted by $E_{\#}(X)$.

Proposition 2. There is an exact sequence

$$
T_{n}(X) \rightarrow \varepsilon_{\sharp}\left(X^{(n)}\right) \rightarrow \varepsilon_{\#}\left(X^{(n-1)}\right),
$$

where $T_{n}(X)$ denotes the kernel of the homomorphism $H^{n}\left(X ; \pi_{n}(X)\right)$ $\rightarrow \operatorname{Hom}\left(\pi_{n}(X), \pi_{n}(X)\right)$.

Note that the map $T_{n}(X) \rightarrow \mathcal{E}_{\#}\left(X^{(n)}\right)$ carries the sum of two cohomology classes into the composition of two homotopy equivalences.

Theorem 3. Let $X$ be a finite CW-complex such that rank $\pi_{i}(X) \leqq 1$ for all $i \leqq \operatorname{dim} X+1$. Then $\varepsilon(X)$ satisfies the maximal condition (i.e., $\mathcal{E}(X)$ and all its subgroups are finitely generated).

Examples of spaces $X$ such that $\varepsilon(X)$ does not satisfy the maximal condition can, for instance, be obtained from Corollary 8 .

Now let $|T|$ stand for the order of the group $T$ and $h_{i}: \pi_{i}(X)$ $\rightarrow H_{i}(X)$ for the Hurewicz homomorphism.

TheOREM 4. Let $X$ be as in Theorem 3. Then $\mathcal{E}(X)$ is finite if the group Hom (coker $\left.h_{i}, \pi_{i}\right)$ is finite for all $i$; in this case

$$
|\varepsilon(X)| \leqq \prod_{i=2}^{\operatorname{dim} X+1} p_{i} \mid \text { Hom }\left(\operatorname{coker} h_{i}, \pi_{i}\right)|| \operatorname{Ext}\left(H_{i-1}, \pi_{i}\right) \mid
$$

where $\pi_{i}=\pi_{i}(X), H_{i}=H_{i}(X)$ and $p_{i}=\mid$ Aut $\pi_{i} \mid$.

As a group defined by composition of maps $\varepsilon(X)$ is generally nonabelian. Theorem 5 gives conditions for $\varepsilon(X)$ to be solvable. If $p$ is a prime we denote by $n\left(H, p^{k}\right)$ the number of times the cyclic group $Z_{p^{k}}$ occurs in the canonical decomposition of the finitely generated abelian group $H$.

Theorem 5. Let $X$ be a finite $\mathrm{CW}$-complex such that

(i) $\operatorname{rank} \pi_{i}(X) \leqq 1$,

(ii) for any $k$

$$
\begin{aligned}
n\left(\pi_{i}(X), p^{k}\right) & \leqq 2 \quad \text { if } p=2,3 \\
& \leqq 1 \quad \text { otherwise }
\end{aligned}
$$

for all $i \leqq \operatorname{dim} X+1$. Then $\&(X)$ is a solvable group.

The proofs of Theorems 3, 4 and 5 proceed similarly. By Lemma 1 an $N$-dimensional complex $X$ can be replaced by its Postnikov section $Y=X^{(N+1)}$. Then in the exact sequence 


$$
1 \rightarrow \varepsilon_{\sharp}(Y) \rightarrow \varepsilon(Y) \stackrel{I}{\rightarrow} \sum_{k} \text { Aut } \pi_{k}(Y)
$$

the group on the right is a finite direct sum. Now one studies $\varepsilon_{\#}(Y)$ and $\sum_{k}$ Aut $\pi_{k}(Y)$ separately. The group $\sum_{k}$ Aut $\pi_{k}(Y)$ is dealt with purely algebraically. On the other hand it follows from Proposition 2 that $\varepsilon_{\sharp}(Y)$ possesses all properties which are shared by finitely generated abelian groups and which carry over to subgroups, factor groups and extensions. This observation immediately yields Theorem 3 . The condition $\operatorname{rank} \pi_{i}(X) \leqq 1$ of Theorem 4 implies that $\mathcal{E}(Y)$ is finite if and only if $\varepsilon_{\#}(Y)$ is finite. By Proposition 2 the latter group is finite if Hom (coker $h_{i}, \pi_{i}$ ) is finite. The assumptions in Theorem 5 together with an argument based on [6, Satz 8] imply that $\sum_{k}$ Aut $\pi_{k}(Y)$ is solvable.

Remarks. (a) Clearly Theorems 3, 4 and 5 also hold for a space with finitely many homotopy groups.

(b) It is easily seen that for a finite complex $X$ the group $\mathcal{E}_{\sharp}(X)$ is a subgroup of $\varepsilon_{\#}\left(X^{(n)}\right)$ for $n>\operatorname{dim} X$. Thus, for instance, $\varepsilon_{\#}(X)$ always is solvable and satisfies the maximal condition.

(c) There are examples of 1-connected finite complexes which show that each of the hypotheses in Theorems 3, 4 and 5 is necessary.

3. Theorems for $H$-spaces. In this section $G$ denotes a 1-connected associative $H$-space of the homotopy type of a finite $\mathrm{CW}$-complex. Let $n_{1}, \cdots, n_{k}$ stand for the dimensions of the algebra generators of the cohomology algebra of $G$ with rational coefficients.

Theorem 6. (a) $\&(G)$ is finitely generated. ${ }^{2}$ (b) $\&(G)$ is a finite group if and only if the $n_{i}$ th Betti number of $G$ equals one for all $i=1, \cdots, k$.

The following theorem shows that for certain $G$ the group $\&(G)$ contains free subgroups of any rank.

THEOREM 7. $\mathcal{E}(G)$ contains a nonabelian free subgroup on at least two generators if and only if rank $\pi_{i}(G)>1$ for some $i$.

COROLlaRy 8. $\&(G)$ satisfies the maximal condition if and only if rank $\pi_{i}(G) \leqq 1$ for all $i$.

The proofs of Theorems 6 and 7 rely on the following considerations. First of all, the collection $\pi(G, G)$ of homotopy classes of basepoint preserving maps from $G$ into itself has the structure of a near-

${ }^{2} \mathrm{~W}$. Shih informs us that he has proved that $\mathcal{E}(Y)$ is finitely generated if $Y$ has finitely many homotopy groups. By our Lemma 1 this implies that $\mathcal{E}(X)$ is finitely generated for any 1-connected finite complex $X$. 
ring [2]. Secondly, it can be shown that the homomorphism $\pi\left(G^{(n)}, G^{(n)}\right) \rightarrow \sum_{k \leqq n} \operatorname{Hom}\left(\pi_{k}(G), \pi_{k}(G)\right)$ is an epimorphism modulo the class of finite abelian groups. In addition in the proof of Theorem 7 one needs the result of Frasch $[4, \S 6]$ that the principal congruence group $\bmod p$ of two-by-two integer matrices is a free group on at least two generators. An important step in the proof of Theorem 6(a) is the application of the following proposition to the ring $\pi\left(G^{(n)}, G^{(n)}\right) / \operatorname{Ker} \Omega$ where $\Omega: \pi\left(G^{(n)}, G^{(n)}\right) \rightarrow \pi\left(\Omega G^{(n)}, \Omega G^{(n)}\right)$ is the loop homomorphism.

Proposition 9. Let $A$ be an associative ring with 1 whose additive group is finitely generated. Then the group of units of $A$ is also finitely generated.

This proposition is a consequence of a result of Borel and HarishChandra [3, Theorem 6.12].

\section{REFERENCES}

1. W. D. Barcus and M. G. Barratt, On the homotopy classification of the extensions of a fixed map, Trans. Amer. Math. Soc. 88 (1958), 57-74.

2. G. Berman and R. J. Silverman, Near-rings, Amer. Math. Monthly 66 (1959), $23-34$.

3. A. Borel and Harish-Chandra, Arithmetic subgroups of algebraic groups. Ann. of Math. (2) 75 (1962), 485-535.

4. H. Frasch, Die Erzeugenden der Hauptkongruenzgruppen für Primzahlstufen, Math. Ann. 108 (1933), 229-252.

5. W. Shih, On the group $E[X]$ of homotopy equivalence maps (to appear).

6. K. Shoda, Ueber die Automorphismen einer endlichen Abelschen Gruppe, Math. Ann. 100 (1928), 674-686.

Princeton University,

The Institute for Advanced Study and

CoRNell UNIVERsity 